\title{
Effect of neutron skin thickness on projectile fragmentation
}

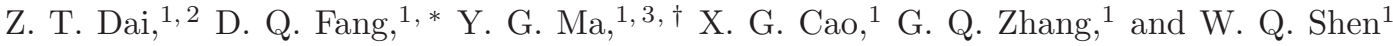 \\ ${ }^{1}$ Shanghai Institute of Applied Physics, Chinese Academy of Sciences, Shanghai 201800, China \\ ${ }^{2}$ University of Chinese Academy of Sciences, Beijing 100049, China \\ ${ }^{3}$ Shanghai Tech University, Shanghai 200031, China
}

(Dated: June 15, 2021)

\begin{abstract}
The fragment production in collisions of ${ }^{48,50} \mathrm{Ca}+{ }^{12} \mathrm{C}$ at $50 \mathrm{MeV} /$ nucleon are simulated via the Isospin-Dependent Quantum Molecular Dynamics (IQMD) model followed by the GEMINI code. By changing the diffuseness parameter of neutron density distribution to obtain different neutron skin size, the effects of neutron skin thickness $\left(\delta_{n p}\right)$ on projectile-like fragments (PLF) are investigated. The sensitivity of isoscaling behavior to neutron skin size is studied, from which it is found that the isoscaling parameter $\alpha$ has a linear dependence on $\delta_{n p}$. A linear dependence between $\delta_{n p}$ and the mean $N / Z[\mathrm{~N}(\mathrm{Z})$ is neutron(proton) number] of PLF is obtained as well. The results show that thicker neutron skin will lead to smaller isoscaling parameter $\alpha$ and $\mathrm{N} / \mathrm{Z}$. Therefore, it may be probable to extract information of neutron skin thickness from isoscaling parameter $\alpha$ and $\mathrm{N} / \mathrm{Z}$.
\end{abstract}

PACS numbers: 21.10.Gv, 24.10.-i, 25.70.Mn

\section{INTRODUCTION}

The neutron skin of a nucleus has been one of the hottest issues in nuclear physics. It is defined as the difference between the neutron and proton root-meansquare $(\mathrm{RMS})$ radii: $\delta_{n p}=\left\langle r_{n}^{2}\right\rangle^{1 / 2}-\left\langle r_{p}^{2}\right\rangle^{1 / 2}$. The mechanism for the formation of neutron skin can be expressed as follows. The proton-neutron interaction is stronger than the proton-proton or neutron-neutron interaction. Therefore, if the neutron number increases for neutron rich nuclei, the mean potential for proton becomes deeper, while the potential for neutron becomes shallower. Consequently, protons are more deeply bound, but neutrons are more loosely bound, which will form the neutron skin [1]. The neutron skin thickness in neutron rich nuclei is crucial for studying the equation of state (EOS) of asymmetric nuclear matter due to its strong correlations with the symmetry energy, the slope and curvature of symmetry energy at the saturation density [2 10]. Thus neutron skin thickness and its effect in nuclear reactions become an important research subject in nuclear physics.

Using the Isospin-Dependent Quantum Molecular Dynamics (IQMD) model, Sun et al. have investigated the neutron to proton ratio $[R(n / p)]$ of emitted fragments from projectile with different neutron skin thickness and shown that there is a strong linear correlation between $R(n / p)$ and $\delta_{n p}$, especially for peripheral collisions [11]. Meanwhile, we have investigated the correlation between the ratio of triton to ${ }^{3} \mathrm{He}\left[R\left(\mathrm{t} /{ }^{3} \mathrm{He}\right)\right]$ and $\delta_{n p}$, which exhibits the similar linear relation [12]. Projectile fragmentation is a well-established technique for rare isotope productions by many radioactive ion beam (RIB) facilities in the world. The projectile fragmentation is used not

\footnotetext{
*Email: dqfang@sinap.ac.cn

${ }^{\dagger}$ Email: ygma@sinap.ac.cn
}

only to study the reaction mechanism of nuclear collision but also to extract some information on fundamental physics [13 15]. The production of heavy fragments will be affected by the neutron and/or proton density distributions of the projectile nuclei. Thus studies on the effect of neutron skin thickness over the projectile-like fragment (PLF) will be very interesting.

In this paper, the relationship between $\delta_{n p}$ and average $N / Z$, the isotope distribution and the yield ratios of PLF will be studied within the framework of IQMD model. The manuscript is organized as follows. In Sec. II, we briefly describe the method, i.e. IQMD model plus GEMINI code. In Sec. III, we present both the results and discussions. Finally, a summary is given in Sec. IV.

\section{THE FRAMEWORK DESCRIPTION}

\section{A. Dynamical model: IQMD}

The quantum-molecular-dynamics (QMD) approach is a many-body theory to describe heavy ion collisions from intermediate to relativistic energies. A general review about the model can be found in Refs. [16, 17]. The IQMD model is based on the QMD model with explicitly inclusion of isospin degrees of freedom. It is well known that the dynamics at intermediate energies is mainly governed by three parts: the mean field, two-body nucleonnucleon collisions and the Pauli blocking. Therefore, it is necessary to include isospin degrees of freedom to the three parts for isospin dependent dynamics. In initialization of the projectile and target nuclei, neutrons and protons should be sampled separately in phase space, especially for nuclei far away from the $\beta$-stability line, of which the neutron and proton density distributions are much different. QMD model has been widely and successfully used in heavy ion collisions [11, 18 29]. Details about the description of the IQMD model can be found in Refs. [11, 12]. 


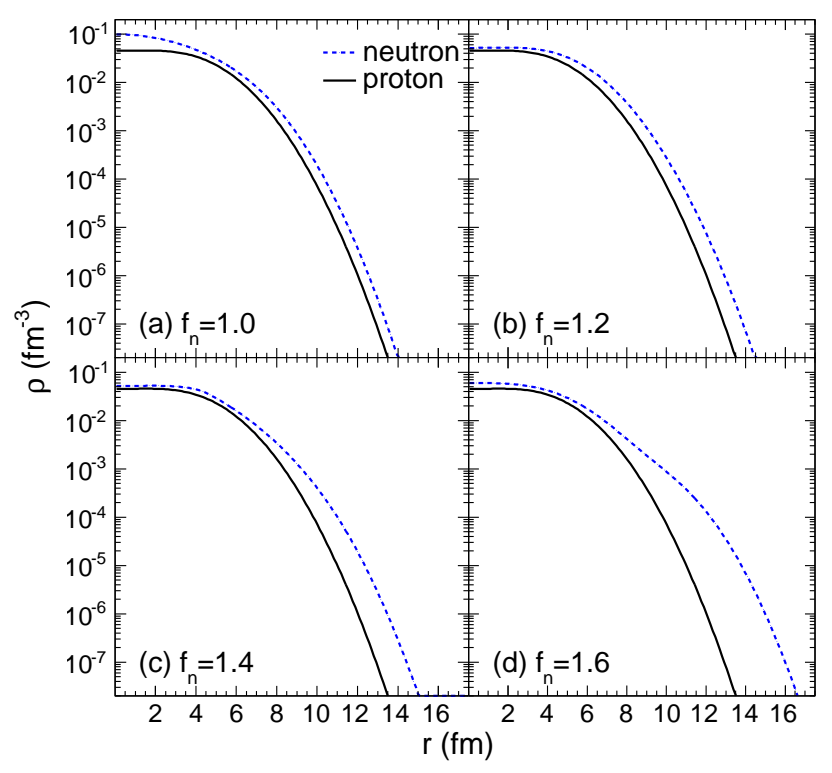

FIG. 1: (Color online) The neutron and proton density profiles calculated from IQMD initialization for ${ }^{50} \mathrm{Ca}$ at $t=0$ with $f_{p}=1.0$ but different $f_{n}$. (a) $f_{n}=1.0\left(\delta_{n p}=0.08 \mathrm{fm}\right)$; (b) $f_{n}=1.2\left(\delta_{n p}=0.21 \mathrm{fm}\right) ;\left(\right.$ c) $f_{n}=1.4\left(\delta_{n p}=0.37 \mathrm{fm}\right)$; (d) $f_{n}=1.6\left(\delta_{n p}=0.54 \mathrm{fm}\right)$.

In the present work, the following potential is used,

$$
\begin{aligned}
U\left(\rho, \tau_{z}\right)=\alpha & \left(\frac{\rho}{\rho_{0}}\right)+\beta\left(\frac{\rho}{\rho_{0}}\right)^{\gamma}+\frac{1}{2}\left(1-\tau_{z}\right) V_{c} \\
& +C_{\text {sym }} \frac{\rho_{n}-\rho_{p}}{\rho_{0}} \tau_{z}+U^{Y u k},
\end{aligned}
$$

where $\rho_{0}=0.16 \mathrm{fm}^{-3}$ is the normal nuclear matter density. $\rho, \rho_{n}$, and $\rho_{p}$ are the total, neutron, and proton densities, respectively. $\tau_{z}$ is the $z$-th component of the isospin degree of freedom, which equals 1 or -1 for neutrons or protons, respectively. The coefficients $\alpha, \beta$ and $\gamma$ are parameters of the nuclear EOS. $C_{\text {sym }}$ is the symmetry energy strength due to the difference between neutron and proton asymmetry in nuclei. In this work, $\alpha=-356 \mathrm{MeV}, \beta=303 \mathrm{MeV}$ and $\gamma=7 / 6$ are taken, which corresponds to the so-called soft EOS with an incompressibility of $K=200 \mathrm{MeV}$ and $C_{\text {sym }}=32 \mathrm{MeV}$. $V_{c}$ is the Coulomb potential and $U^{Y u k}$ is the Yukawa potential. Many theoretical studies show a strong correlation between symmetry energy and neutron skin size, while there is still large uncertainty of neutron skin size and symmetry energy [30, 31]. As discussed in the reference 32, both the symmetry and neutron skin have effects on the particle productions. Therefore, it is interesting to study the effects of neutron skin and symmetry energy separately. The correlations between symmetry energy and the particle or fragment productions have also been investigated in many theoretical studies 32 34]. The flexibility of adjusting independently the size of neutron skin of colliding nuclei is useful for our analyses in this work. Moreover, in this work, different neutron skin thicknesses mainly influence the neutron density distribution in the surface region of nuclei, so our study is focused on the peripheral collisions. In the peripheral collisions, the effect of neutron skin is larger than the effect due to the symmetry energy [32].

In the initialization of IQMD, the density distributions of neutrons and protons are assumed to follow the Fermitype form according to the droplet model [35, 36],

$$
\rho_{i}(r)=\frac{\rho_{i}^{0}}{1+\exp \left(\frac{r-C_{i}}{f_{i} t_{i} / 4.4}\right)}, \quad i=n, p,
$$

where $\rho_{i}^{0}$ is the normalization constant which ensures that the integration of the density distribution equals to the number of neutrons $(i=\mathrm{n})$ or protons $(i=\mathrm{p}) ; t_{i}$ is the diffuseness parameter; $C_{i}$ is the half density radius of neutron or proton determined by the droplet model [36].

$$
C_{i}=R_{i}\left[1-\left(b_{i} / R_{i}\right)^{2}\right], \quad i=n, p,
$$

here $b_{i}=0.413 f_{i} t_{i}, R_{i}$ is the equivalent sharp surface radius of neutron or proton. $R_{i}$ and $t_{i}$ are given by the droplet model. The factor $f_{i}$ is used to adjust the diffuseness of density distribution. In this work, $f_{p}=1.0$ is used in Eq.(2) for the proton density distribution, while $f_{n}$ in Eq.(2) is varied from 1.0 to 1.6 for the neutronrich projectile. Different values of $\delta_{n p}$ can be deduced from Eq.(2) by changing $f_{n}$. Using the density distributions given by the droplet model, initial coordinates of nucleons in the nucleus are sampled in terms of the Monte Carlo method. After initialization, the samples with satisfactory stability and expected neutron skin size will be selected as candidates for collisions, as described in Refs. 11, 12]. Fig. 1 shows the neutron and proton density profiles of ${ }^{50} \mathrm{Ca}$ calculated from IQMD initialization. In the simulation, $f_{n}$ is $1.0,1.2,1.4,1.6$ respectively. It can be seen that with the increase of $f_{n}$, the neutron density distribution becomes more extended, while that of proton is almost same in the four cases. The related neutron skin thickness are $0.08 \mathrm{fm}, 0.21 \mathrm{fm}, 0.37 \mathrm{fm}$ and $0.54 \mathrm{fm}$, respectively. The initial neutron skin can last a long time comparable with the reaction time, although a small-amplitude oscillation is visible. This level of stability of the initial samples is good enough for the purpose of this study. As a consequence, we can study the neutron skin effect on the production of fragments with these samples. In this work, the fragments are constructed by a coalescence model, in which nucleons with relative momentum smaller than $\mathrm{P}_{0}=300 \mathrm{MeV} / \mathrm{c}$ and relative distance smaller than $\mathrm{R}_{0}=3.5 \mathrm{fm}$ will be identified as a cluster.

\section{B. Decay model: GEMINI}

The pre-fragments produced in IQMD are excited which are not comparable with the final products measured experimentally, it is necessary to take into account 


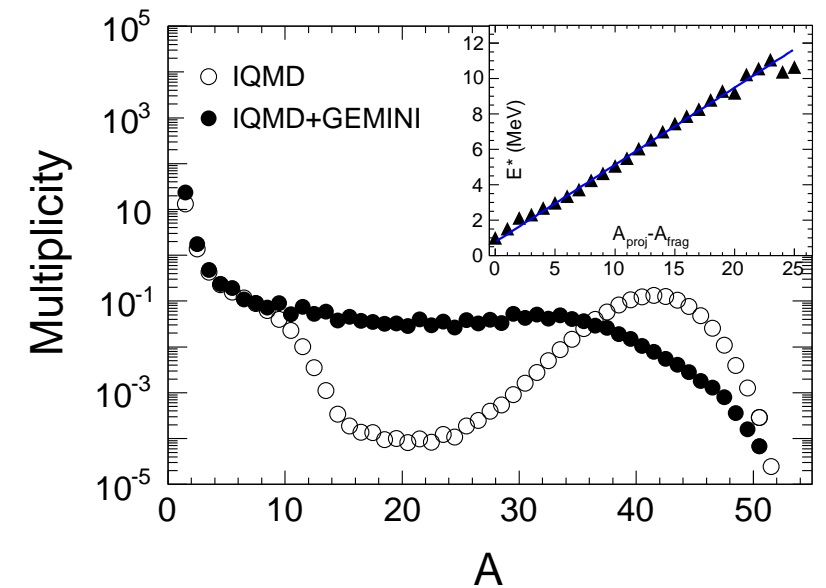

FIG. 2: Fragment mass multiplicity distribution in $50 \mathrm{MeV} /$ nucleon ${ }^{50} \mathrm{Ca}+{ }^{12} \mathrm{C}$ with $f_{n}=1.0$ and $0.6<b / b_{\max }<$ 1.0. Open circles represent IQMD calculation at $t=200 \mathrm{fm} / \mathrm{c}$ and solid points are results after the evaporation by GEMINI. The excitation energy per nucleon of the prefragment as a function of $A_{\text {proj }}-A_{\text {frag }}$ calculated by IQMD simulations is shown in the inset.

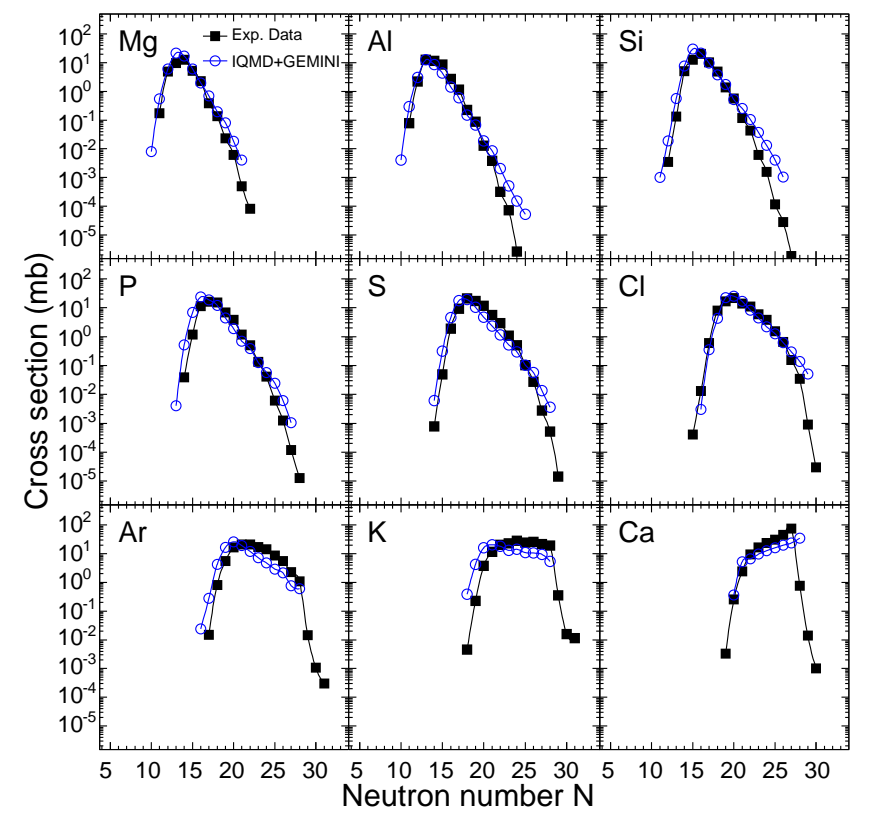

FIG. 3: (Color online) The isotopic distributions plotted as a function of neutron number $\mathrm{N}$ for elements with $12 \leq Z \leq 20$ in $140 \mathrm{MeV} /$ nucleon ${ }^{48} \mathrm{Ca}+{ }^{9} \mathrm{Be}$. Solid squares are the experimental data taken from Ref. [47], and open circles are the calculated results with IQMD plus GEMINI.

the evaporation effect to obtain realistic results. The GEMINI code is used to calculate de-excitation of these fragments 37]. GEMINI is a Monte Carlo code which allows not just light-particle evaporation and symmetric fission, but all possible binary-decay modes. It deexcites a source nucleus by a series of sequential binary decays until the excitation energy of the excited fragment being unable to go further decay. By using GEMINI, the fragment productions are widely and successfully investigated with QMD model [38 40], as well as, antisymmetrized molecular dynamics (AMD) model [41, 42]. In IQMD, we can construct the angular momentum and excitation energy for each fragment which are used as input to GEMINI. The angular momentum of each nucleon in the fragment is calculated according to classical mechanics

$$
\overrightarrow{L_{i}}=\overrightarrow{R_{i}} \times \vec{P}_{i}
$$

where $\vec{R}_{i}$ and $\vec{P}_{i}$ are coordinate and momentum vector of the $i$-th nucleon of the fragment in the center of mass (CM) frame of the fragment. The total angular momentum of the fragment is the summation of Eq. (4) over all nucleons in it. The excitation energy of the primary fragment is calculated by following equation,

$$
E^{*}=E_{\text {bind }}^{\text {excited }}-E_{\text {bind }}^{\text {ground }}
$$

where $E_{\text {bind }}^{\text {excited }}$ is the binding energy of the excited fragment calculated from IQMD, and $E_{\text {bind }}^{\text {ground }}$ is the binding energy of the ground state taken from nuclear mass table [46]. The inset of Fig. 2 displays $E^{*}$ as a function of $A_{\text {proj }}-A_{\text {frag }}$, where $A$ is mass number and the index proj and frag refer to the projectile and fragment, respectively. Although the constructed excitation energy is model dependent, the excitation energy obtained from our IQMD calculation is comparable with that constructed from experimental data and model simulations [43 45].

With the calculated excitation energy and angular momentum, the fragments at $t=200 \mathrm{fm} / \mathrm{c}$ will be deexcited by using GEMINI. Since the main purpose of the present work is to study the effect of neutron skin thickness of the projectile on the production of heavy fragments, the calculations are focused on peripheral collisions. The reduced impact parameter is used to describe the centrality of collision which is defined as $b / b_{\max }$, with $b_{\max }$ being the maximum impact parameter. For peripheral reaction, the nucleon-nucleon interaction on the nuclear surface plays an important role, where nucleons are loosely bound and the density distributions are quite different for neutron and proton. Fig. 2 represents the comparison of mass versus multiplicity of fragments with and without GEMINI decay under the condition of $0.6<b / b_{\max }<1.0$. In the peripheral collision of IQMD, the main reaction mechanism of fragment production is abrasion and evaporation of nucleons or light clusters, which leads to the excess of heavy products and deficiency of intermediate mass fragments (IMFs). In contrast, applying the afterburner significantly improves the fragments production since more complex fragments are emitted in the de-excitation calculation. 


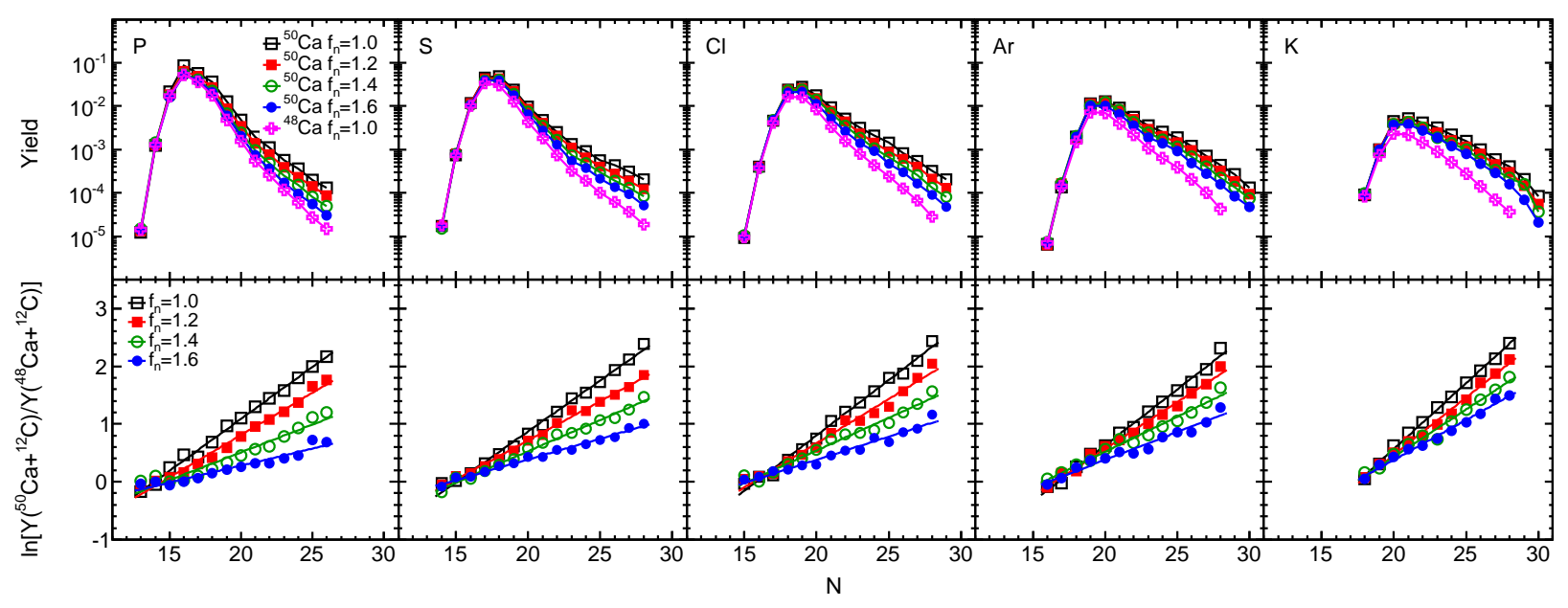

FIG. 4: (Color online) The isotopic yield per event plotted as function of the neutron number $N$ with the charge number of fragment varying from 15 to 19 in ${ }^{50} \mathrm{Ca}+{ }^{12} \mathrm{C}$ and ${ }^{48} \mathrm{Ca}+{ }^{12} \mathrm{C}$ at $50 \mathrm{MeV} /$ nucleon (upper row). The related isotopic yield ratios of the two reactions as function of $N$ is displayed in the lower row. In the calculations, $f_{n}$ is varied from 1.0 to 1.6 for ${ }^{50} \mathrm{Ca}+{ }^{12} \mathrm{C}$, while it is 1.0 for ${ }^{48} \mathrm{Ca}+{ }^{12} \mathrm{C}$.

\section{RESULTS AND DISCUSSIONS}

Before investigating the neutron skin effect in fragment production, the isotopic distributions calculated by IQMD plus GEMINI are compared with experimental data to evaluate the validity of the present method. In Fig. 3 the measured cross sections from Ref. 47] and the calculated results of IQMD plus GEMINI for fragments with $12 \leq Z \leq 20$ from ${ }^{48} \mathrm{Ca}+{ }^{9} \mathrm{Be}$ at $140 \mathrm{MeV} /$ nucleon are plotted. The calculated yields are scaled to the experimental data for each isotope separately. From this comparison, we can see that our calculations can reproduce the shape of the isotopic distribution data quite well, which suggests that the IQMD plus GEMINI model is reasonable for calculating the heavy fragments. This is also demonstrated in Refs. 38, 40]. Meanwhile, Mock et al. have well reproduced this experimental data with heavy ion phase space exploration (HIPSE), AbrasionAblation (AA) and AMD 44, 45].

As discussed in Ref. [48], the neutron skin plays a significant role in the production of fragment, especially for the production of neutron-rich nuclei. Hence, to reveal the correlation between neutron skin and the fragment production is interesting. Using the IQMD model, collisions of ${ }^{48,50} \mathrm{Ca}$ projectile on ${ }^{12} \mathrm{C}$ target at $50 \mathrm{MeV} /$ nucleon are simulated. In our simulations, the diffuseness parameter $f_{n}=1.0$ is used for ${ }^{48} \mathrm{Ca}$, while $f_{n}$ varies from 1.0 to 1.6 for ${ }^{50} \mathrm{Ca}$. In the upper row of Fig. 4. the production yield per event for five isotopes with the charge number varying from 15 to 19 are plotted. The reason why we choose the fragments with the charge number varying from 15 to 19 is explained as follows. The main purpose of this work is to study the neutron skin effects on fragments. In our IQMD, probability of fusion is small, so the fragments with charge number larger than projectile are neglected. The lighter fragments, which reach chemical balance at the end of the reaction will keep little information of the source nuclei, so the effect of neutron skin thickness on these fragments is very small. While the heavy residue can keep as much information of the projectile as possible. Therefore, fragments with the charge number varying from 15 to 19 are chosen as a probe of neutron skin. Firstly, we compare the fragment isotopic distributions from reactions induced by projectiles with different neutron excess. By comparing the two systems ${ }^{48} \mathrm{Ca}+{ }^{12} \mathrm{C}$ and ${ }^{50} \mathrm{Ca}+{ }^{12} \mathrm{C}$ with $f_{n}=1.0$, it is demonstrated that there are more neutron-rich fragments produced with increasing the neutron excess of the projectile. This result is similar to that in Ref. [13]. Secondly, we compare the reactions ${ }^{50} \mathrm{Ca}+{ }^{12} \mathrm{C}$ with different $f_{n}$, which relates to different $\delta_{n p}$. With the increase of neutron skin thickness, yields of the isotope distributions will decrease in the neutron-rich side but have almost no change in the neutron-deficient side, which is consistent with the results by statistical abrasion-ablation model 49].

Meanwhile, it has been shown that the yield ratios of isotopic fragments from two similar reactions that differ only in the isospin asymmetry follow scaling laws $[50-56]$. The isotope yield ratios $R_{21}(N, Z)=Y_{2}(N, Z) / Y_{1}(N, Z)$ measured in two different nuclear reactions, 1 and 2 , are found to exhibit an exponential relationship as a function of the neutron number $\mathrm{N}$ and proton number $\mathrm{Z}$ [50], which can be expressed by following formula:

$$
R_{21}(N, Z)=\frac{Y_{2}(N, Z)}{Y_{1}(N, Z)}=C \exp (\alpha N+\beta Z)
$$

where $\alpha$ and $\beta$ are two scaling parameters and $C$ is an overall normalization constant.

The isoscaling phenomenon is systematically investi- 


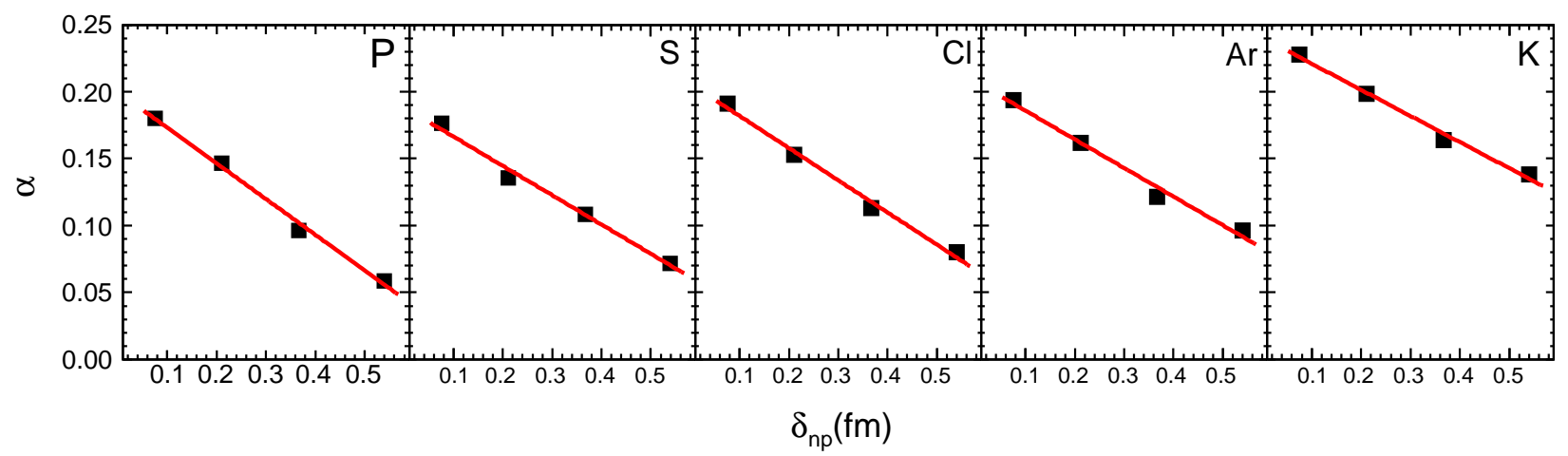

FIG. 5: (Color online) The isoscaling parameter $\alpha$ as a function of $\delta_{n p}$ for fragments with the charge number varying from 15 to 19 in $50 \mathrm{MeV} /$ nucleon ${ }^{50} \mathrm{Ca}+{ }^{12} \mathrm{C}$.

gated for fragments not heavier than $Z=8$. However, it would be interesting to investigate the behavior of heavier fragments up to the region of PLF, because the heavy residues, which is mainly produced by abrasion and evaporating mechanism, may preserve some memory of the source configuration, for example, the neutron and proton density distribution. The isotopic scaling of heavy projectile residues is observed in both experiment and theoretical simulation [54 58]. Consequently, from the research of isoscaling of heavy residues of projectile, some information about neutron skin of neutron-rich projectile could be extracted, since the neutron skin does effect the production of fragments [48]. This is our new start point. In order to investigate the neutron skin size effect on isoscaling, the isotopic yield ratios between two reactions ${ }^{50} \mathrm{Ca}+{ }^{12} \mathrm{C}$ and ${ }^{48} \mathrm{Ca}+{ }^{12} \mathrm{C}$ are plotted as a function of the fragment neutron number, as shown in the lower row of Fig. 4 In the calculation, $f_{n}=1.0$ is used for ${ }^{48} \mathrm{Ca}$, while $f_{n}=1.0,1.2,1.4$ and 1.6 are used for ${ }^{50} \mathrm{Ca}$. From the figure, we can see that larger neutron skin size will suppress

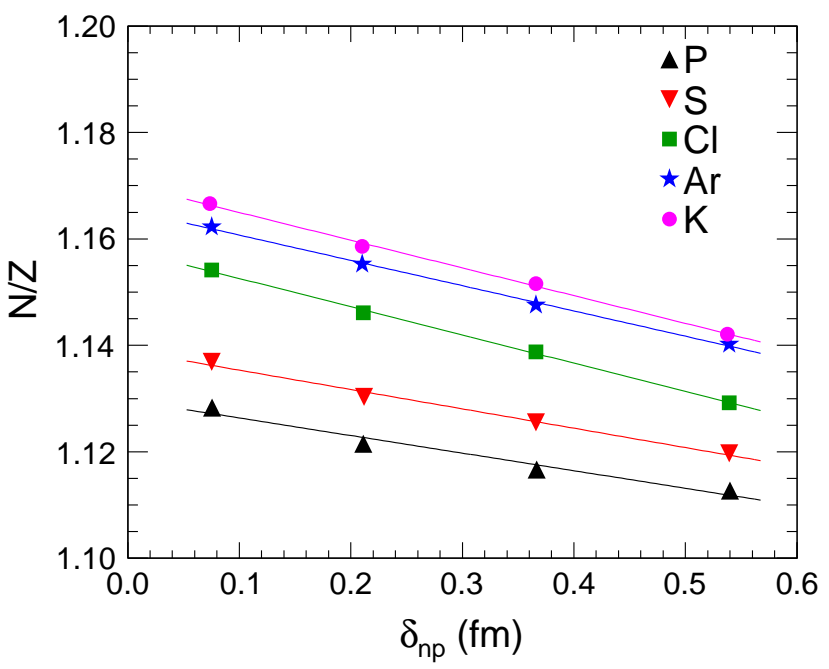

FIG. 6: (Color online) The average $N / Z$ of fragment as a function of $\delta_{n p}$ in $50 \mathrm{MeV} /$ nucleon ${ }^{50} \mathrm{Ca}+{ }^{12} \mathrm{C}$. the production of the neutron-rich fragment. Although the neutron number and proton number of projectiles and targets are not changed in reactions ${ }^{50} \mathrm{Ca}+{ }^{12} \mathrm{C}$ and ${ }^{48} \mathrm{Ca}+{ }^{12} \mathrm{C}$, the neutron density of ${ }^{50} \mathrm{Ca}$ is different with different $f_{n}$. Eq.(6) can be simplified for isotopic yield ratio with the same charge number,

$$
R_{21}(N)=C^{\prime} \exp (\alpha N) .
$$

In the grand-canonical approximation, the scaling parameter $\alpha$ is equal to the difference of the chemical potentials for neutrons in the two systems, $\alpha=\mu_{n} / T$ [50, 51]. The parameter $\alpha$ is extracted by fitting $\ln \left[R_{21}(N, Z)\right]$ as a function of $N$. $\alpha$ dependence on $\delta_{n p}$ for isotopes with proton number varying from $Z=15$ to $Z=19$ is displayed in Fig. 5 . One can see that $\alpha$ decreases linearly with the increasing of neutron skin thickness in the projectile. It is indicated that the scaling parameter dose not keep constant for the heavy residues with proton number close to the projectile, which is different from that for light fragment. This different isoscaling behavior between heavy and light fragments may result from different formation dynamics. The light fragments are mainly produced from multi-fragmentation, in which the system has reached chemical balance, which leads to the similar isoscaling behavior of light fragment. While the heavy residues close to projectile originate from nucleons abrasion or evaporation. In this case, most of the nucleon in projectile may only act as an spectator, and accordingly, the system is not balanced. Nevertheless, the heavy residues will keep a memory of the projectile to some degree. From this result some information of neutron skin could be extracted by measuring the isoscaling behavior of the heavy residues.

Finally, neutron skin effect on $N / Z$ of heavy PLF is also investigated. The average $N / Z$ for PLF with charge number from $Z=15$ to $Z=19$ as a function of $\delta_{n p}$ is plotted in Fig. 6. It displays that $N / Z$ decreases with the increasing of $\delta_{n p}$, and there is a good linear correlation between $N / Z$ and $\delta_{n p}$. This relationship could be regard as another probe for neutron skin thickness. We also can see that for a certain $\delta_{n p}$, the $N / Z$ will increase with 
the increasing of charge number, which is similar to the results in Ref. [58]

The neutron skin effect on fragment production, isoscaling, and $N / Z$ of residues could be explained as following. For different neutron skin thickness, the mainly difference of neutron density distributions is in the surface region. With increasing of $\delta_{n p}$, more neutrons are pushed to the surface of nuclei. Consequently, more neutrons will be abraded in peripheral collision, which makes the residue less neutron-rich. Large $\delta_{n p}$ makes the neutron become more loosely bound, and more neutrons will be evaporated.

\section{SUMMARY}

Using the isospin dependent quantum molecular (IQMD) model followed by GEMINI, the neutron skin effect on the production of projectile-like fragments are investigated in peripheral collisions of $50 \mathrm{MeV} /$ nucleon ${ }^{50} \mathrm{Ca}+{ }^{12} \mathrm{C}$ and ${ }^{48} \mathrm{Ca}+{ }^{12} \mathrm{C}$. By changing the neutron diffuseness parameter of ${ }^{50} \mathrm{Ca}$ to obtain different neutron skin thickness $\left(\delta_{n p}\right)$, the dependence of isotopic distributions, neutron to proton ratio $(N / Z)$, and isoscaling behavior of PLF on $\delta_{n p}$ are studied. It is demonstrated that larger $\delta_{n p}$ suppresses the production of neutron-rich
PLF. This is because projectile with larger $\delta_{n p}$ prefers to produce more free neutrons and neutron-rich light clusters. The isoscaling behavior in ${ }^{50} \mathrm{Ca}+{ }^{12} \mathrm{C}$ and ${ }^{48} \mathrm{Ca}+{ }^{12} \mathrm{C}$ are also investigated. The extracted isoscaling parameter $\alpha$ decreases linearly with the increase of $\delta_{n p}$. The dependence of neutron to proton ratio $(N / Z)$ of PLF on $\delta_{n p}$ displays the similar trend. With the increase of $\delta_{n p}$, more neutrons will distribute in the nuclear surface. Consequently, they will be abraded in peripheral collision more easily. In conclusion, the isotopic distributions, isoscaling parameter $\alpha$, and neutron to proton ratios $N / Z$ of PLF have dependence on $\delta_{n p}$. This dependence could probably be used to extract some information of neutron skin from experiments.

\section{Acknowledgements}

This work was supported in part by the Major State Basic Research Development Program in China under Contract No. 2013CB834405, the National Natural Science Foundation of China under Contract No.s 11175231, 11035009, 11475244, 11421505 and 11205079 and the Knowledge Innovation Project of Chinese Academy of Science under Grant No. KJCX2-EW-N01.
[1] N. Fukunishi, T. Otsuka, I. Tanihata, Phys. Rev. C 48, 1648 (1993).

[2] B. A. Brown, Phys. Rev. Lett. 85, 5296 (2000).

[3] C. J. Horowitz and J . Piekarewicz, Phys. Rev. Lett. 86, 5647 (2001).

[4] C. J. Horowitz and J . Piekarewicz, Phys. Rev. C 66, 055803 (2002).

[5] S. Typel and B. A. Brown, Phys. Rev. C 64, 027302 (2001).

[6] R. J. Furnstahl, Nucl. Phys. A 706, 85 (2002).

[7] S. Karataglidis, K. Amos, B . A. Brown, and P. K. Deb, Phys. Rev. C 65, 044306 (2002).

[8] M. Centelles, X. Roca-Maza, X. Viñas, and M. Warda, Rev. Lett. 102, 122502 (2009).

[9] A. Carbone, G. Colò, A. Bracco et al., Phys. Rev. C 81, 041301(R) (2010).

[10] F. J. Fattoyev and J. Piekarewicz, Rev. Lett. 111, 162501 (2013).

[11] X. Y. Sun, D. Q. Fang, Y. G. Ma et al., Phys. Lett. B 682, 396 (2010).

[12] Z. T. Dai, D. Q. Fang, Y. G. Ma, et al., Phys. Rev. C. 89, 014613 (2014).

[13] D. Q. Fang, W. Q. Shen, et al., Phys. Rev. C 61, 044610 (2000).

[14] Y. G. Ma, Q. M. Su, W. Q. Shen, et al., Phys. Rev. C 60, 024607 (1999).

[15] S. Lukyanov, M. Mocko, et al., Phys. Rev. C 80, 014609 (2009).

[16] J. Aichelin, A. Rosenhauer, G. Peilert, H. Stöcker, W. Greiner, Phys. Rev. Lett. 58, 1926 (1987).

[17] C. Hartnack, R. K. Puri, J. Aichelin, J. Konopka, S. A.
Bass, H. Stöcker, and W. Greiner, Eur. Phys. J. A 1, 151 (1998).

[18] X. G. Cao et al., Phys. Rev. C 86, 044620 (2012).

[19] J. Y. Liu et al., Phys. Lett. B 617, 24 (2005).

[20] C. Tao, Y. G. Ma et al., Phys. Rev. C 87, 014621 (2013); Nucl. Sci. Tech. 24, 030502 (2013); J. Wang et al., Nucl. Sci. Tech. 24, 030501 (2013).

[21] Y. G. Ma, W. Q. Shen, Phys. Rev. C 51, 710 (1995).

[22] F. S. Zhang, L. W. Chen, Z. Y. Ming, Z. Y. Zhu, Phys. Rev. C 60, 064604 (1999).

[23] Y. G. Ma, W. Q. Shen, Phys. Rev. C 51, 3256 (1995).

[24] Y. G. Ma, Y. B. Wei, et al., Phys. Rev. C 73, 014604 (2006).

[25] C. L. Zhou, Y. G. Ma, D. Q. Fang, G. Q. Zhang, Phys. Rev. C 88, 024604 (2013).

[26] Y. X. Zhang, L. Z. Xiaet al., Nucl. Sci. Tech. 24, 050503 (2013).

[27] Z. Q. Feng, Nucl. Sci. Tech. 24, 050504 (2013).

[28] S. Kummar, Y. G. Ma, Nucl. Sci. Tech. 24, 050509 (2013).

[29] C. C. Guo, J. Su, F. S. Zhang, Nucl. Sci. Tech. 24, 050513 (2013).

[30] B. K. Agrawal, J. N. De et al et al., Phys. Rev. Lett. 109, 262501 (2012).

[31] M. B. Tsang, Y. X. Zhang, Phys. Rev. Lett. 102, 122701 (2009).

[32] G. F. Wei, B. A. Li et al., Phys. Rev. C 90, 014610 (2014).

[33] Sanjeev Kumar, Y. G. Ma et al., Phys. Rev. C 85, 0024620 (2012).

[34] J. Pu, J. H. Chen, Sanjeev Kumar et al., Phys. Rev. C 87, 047603 (2013). 
[35] W. D. Myers and W. J. Swiatecki, Nucl. Phys. A 336, 267 (1980).

[36] W. D. Myers, K. H. Schmidt, Nucl. Phys. A 410, 61 (1983).

[37] R. J. Charity et al., Nucl. Phys. A 483, 371 (1988).

[38] K. Hagel, M. Gonin, R. Wada et al., Phys. Rev. C 50, 4 (1994).

[39] W. D. Tian, Y. G. Ma, X. Z. Cai et al., Int. J. Mod. Phys. E 17, 9 (1994).

[40] J. Su, F. S. Zhang et al., Phys. Rev. C 84, 037601 (2011).

[41] Y. G. Ma, R. Wada, K. Hagel et al., Phys. Rev. C 65, 051602(R) (2002).

[42] M. Huang, Z. Chen, S. Kowalski, Y. G. Ma et al., Phys. Rev. C 81, 044620(2010).

[43] D. Theriault, A. Vallee et al., Phys. Rev. C 71, 014610 (2005).

[44] M. Mocko, M. B. Tsang, D. Lacroix et al., Phys. Rev. C 78, 014612 (2008).

[45] M. Mocko,Ph.D. thesis, Michigan State University, 2006.

[46] G. Audi, A. H. Wapstra, Nucl. Phys. A 729, 337 (2003).

[47] M. Mocko, M. B. Tsang, L. Andronenko et al., Phys. Rev. C 74, 054612 (2006).
[48] G. A. Souliotis, M. Veselsky, G. Chubarian et al., Phys. Rev. Lett 91, 2 (2003).

[49] C. W. Ma, S. S. Wang, Chin. Phys. C 35, 11 (2011).

[50] M. B. Tsang, W. A. Friedman, C.K. Gelbke et al., Phys. Rev. Lett 86, 22 (2001).

[51] A. S. Botvina, O. V. Lozhkin, and W. Trautmann, Phys. Rev. C 65, 044610 (2002).

[52] W. D. Tian, Y. G. Ma, X. Z. Cai, D. Q. Fang et al., Phys. Rev. C 76, 024607 (2007).

[53] M. Veselsky, G. A. Souliotis and S. J. Yennello, Phys. Rev. C 69, 031602(R) (2004).

[54] Y. Fu, D. Q. Fang, Y. G. Ma et al., Nucl. Phys. A 834, 584 (2010).

[55] G.A. Souliotis, M.Veselsky, D.V. Shetty, S.J.Yennello, Phys. Lett. B 588, 35-42 (2004).

[56] M. Huang, Z.Chen, S.Kowalski et al., Nucl. Phys. A 847, 233-242 (2010).

[57] Z. Chen, S. Kowalski, M. Huang,R. Wadaet al., Phys. Rev. C 81, 064613 (2010).

[58] G. A. Souliotis, D. V. Shetty, M. Veselsky et al., Phys. Rev. C 68, 024605 (2003); 